\title{
La divulgación de la ciencia en el siglo 21
}

\section{The Divulgation of Science in the 21st Century}

\author{
Rubí Estela Morales Salas \\ Universidad de Guadalajara \\ https://orcid.org/0000-0003-4133-4712 \\ rubi.morales@suv.udg.mx \\ México
}

\section{Resumen:}

La ciencia es producto de los esfuerzos del ser humano para sistematizar el conocimiento y se remonta a tiempos prehistóricos cuando las civilizaciones del neolítico tuvieron la necesidad de resolver preguntas cotidianas que ayudaban a mejorar su calidad de vida. Con el paso del tiempo no bastó con sistematizar el conocimiento, sino que fue necesario comunicarlo, difundirlo y divulgarlo. Esta comunicación expone el devenir de la divulgación de la ciencia y las demandas que tiene en el siglo XXI al hacer uso de las TIC. Se concluye que divulgar la ciencia va más allá de solo comunicar, sino que, debe ser factible de penetrar en el entendimiento del público en general para que se pueda transformar la realidad social, apoyando estas acciones a través del uso y aplicación del poder derivado de las TIC.

Palabras clave: Divulgar; difundir; ciencia; redes sociales; TIC.

\begin{abstract}
:
Science is the product of human efforts to systematize knowledge and it dates back to prehistoric times, when Neolithic civilizations needed to solve everyday questions that helped improve their quality of life. With the passage of time it was not enough to systematize knowledge, but it was necessary to communicate and disseminate it. This communication exposes the evolution of the divulgation of science and the needs it has in the 21st century when making use of ICT. It is concluded that science's divulgation goes beyond just communicating but must be feasible to penetrate the understanding of the general public so that social reality can be transformed, supporting these actions through the use and application of the power derived from ICT.
\end{abstract}

Keywords: Divulgation; science; social networks; ICT.

Recibido: 01/03/2021 | Aceptado: 14/06/2021 | Publicado: 07/07/2021

| pág. 133 - 151 
La divulgación de la ciencia en el siglo 21

\section{| Introducción}

La ciencia es producto de los esfuerzos del ser humano para sistematizar el conocimiento y data de tiempos prehistóricos cuando las civilizaciones del neolítico tuvieron la necesidad de resolver preguntas cotidianas que ayudaban a mejorar su calidad de vida. El solo hecho de observar y tratar de entender a la naturaleza por parte de las civilizaciones antiguas, ha sido considerado como los inicios de la ciencia. Estos hechos, por lo general, estaban influenciados por la religión, la mitología, la magia o el misticismo. Conforme fue evolucionando el ser humano, la ciencia cobró vital importancia en las esferas de la sociedad.

No se puede precisar el origen de la ciencia, ya que, "el comienzo histórico exacto de la ciencia es indeterminable en el tiempo. Se plantea que su surgimiento tiene lugar en el momento donde se descubre (o se establece) la relación de que unos fenómenos son causa y otros efectos" (Academia de Ciencias de la Unión de Repúblicas Socialistas Soviéticas [URSS], 1977, citado en Cañedo Andalia, 1996, párr. 1).

Un primer encuentro de la ciencia como tal, se remonta a la antigua Grecia, aproximadamente en los años 300 a. C., donde los filósofos que en esa época proliferaron, comenzaron a separar la comprensión de la naturaleza del pensamiento religioso y mítico que, en la mayoría de los casos, las personas solían confundir con hechos mágicos o de misticismo. Así, los filósofos griegos comenzaron a crear distintas disciplinas que ayudaban a diferenciar lo real de lo mágico, como la lógica, la matemática, la física, la geometría y la biología.

Desde entonces, la ciencia cobra otro sentido y evoluciona no solo en su concepto, sino también en el alcance 
y las formas para comunicarla, apoyadas por múltiples medios como lo son las Tecnologías de la Información y Comunicación (TIC); la difusión y divulgación pone a la ciencia al alcance de todo tipo de público. Esta comunicación expone el devenir de la divulgación de la ciencia y las demandas que tiene en el siglo XXI al hacer uso de las TIC.

\section{| Un poco de historia}

"La palabra ciencia deriva del latín scientia, que significa 'conocimiento' o 'saber' y como tal, no se restringe a un área específica” (Ciencia, 2020, párr. 4). En este sentido, la ciencia no se limita a una sola disciplina o campo de conocimiento, sino que comprende a las ciencias formales, ciencias sociales y humanas, ciencias naturales, entre otras; las cuales favorecen al desarrollo de teorías y métodos para cada área.

Dicho lo anterior y tomando en cuenta la actualidad, de acuerdo con la Real Academia Española ([RAE], 2021a), se entiende por ciencia el "conjunto de conocimientos obtenidos mediante la observación y el razonamiento, sistemáticamente estructurados $\mathrm{y}$ de los que se deducen principios $\mathrm{y}$ leyes generales con capacidad predictiva y comprobables experimentalmente” (párr. 1). Se puede decir; que el propósito de la ciencia es observar los fenómenos que ocurren en la naturaleza y describirlos mediante leyes que retraten su realidad, de tal forma que se llegue a la comprensión y explicación científica, a fin de mejorar el entorno de los seres humanos.

En este sentido, observar para descubrir ya no fue suficiente; se pasó a un nivel de comprensión. Sin embargo, entre más evolucionaba el raciocinio del ser humano, mayor necesidad sentía de conocer de manera más profunda el mundo que lo rodeaba. Se transitó entonces, a un nivel de conciencia más elevado que demandaba la explicación, análisis y evaluación de los fenómenos; es así que el humano empezó a empoderarse, gracias al conocimiento que se iba ganando a través del desarrollo de teorías que coadyuvaron a la evolución del hombre y de las cosas para el hombre.

Se aprecia la evolución que ha tenido el concepto de ciencia, pero no solo 
su significado se ha ido modificando al pasar de los siglos, sino también, la forma en que se comunica o se transmite de generación en generación. La aplicación de normas y criterios verificables a través del método científico, basados en la observación, formulación de hipótesis, experimentación y demostración, debía comunicarse al mundo; es entonces que comienza la divulgación de la ciencia.

Se tienen registros sobre los primeros hallazgos realizados para comunicar la ciencia, los cuales datan del siglo XVII, cuando en el año de 1612 el astrónomo italiano Galileo Galilei, hizo una publicación que refería a las manchas solares, confesándole "a su amigo el canónigo Paolo Gualdo, la he escrito en idioma vulgar porque he querido que toda persona pueda leerla” (Núñez, 2010, párr. 1). Al referirse al idioma vulgar, significa que la escribió en italiano y no en latín (lengua oficial de los primeros romanos que data del siglo III a. C).

El latín como idioma prevalecía aún en el siglo XVII, en la administración, en la política y en la religión y solía ser usado por la élite. Durante la época del Renacimiento, la lengua italiana (como la conocemos hoy) se impuso sobre el latín tanto hablado como escrito y es entonces que autores como Dante Alighieri, Giovanni Boccaccio y Francesco Petrarca comenzaron a escribir sus obras en italiano. Con su obra La Divina Comedia, es que Dante Alighieri se convierte en el auténtico precursor del nacimiento del italiano; ya que este escritor florentino, al igual que Galilei, quería que su obra la entendiera la población en general y no solo un número reducido que pertenecía a la élite italiana.

Tras la publicación de Galilei sobre las manchas solares, siguieron otras más, entre ellas su obra cumbre denominada "Diálogo sobre los dos sistemas del mundo", publicada en el año de 1632. En ésta, además de hacer una demostración magistral de la dialéctica y retórica discursiva, nos presenta un ejemplo sobre el uso de los diálogos como útil vehículo de divulgación, ya que era descrita con un carácter flexible, vulgar, irónico $y$ puede decirse que un tanto divertido. Esta obra fue condenada por la Santa Inquisición; sin 
embargo, la valentía “de publicar en un idioma común, marcó un camino que sería adoptado por otros, como René Descartes, quien en 1637 publicó en francés su Discurso del Método, y Robert Boyle, que dio a la luz en inglés en 1661, El químico escéptico" (Núñez, 2010, párr. 4).

\section{| Diferencia entre divulgación y difusión de la ciencia}

La difusión y divulgación son términos que se encuentran muy vinculados en sus significados, de acuerdo con la RAE (2021b) divulgar significa "publicar, extender, poner al alcance del público algo" (párr.1). Mientras que difundir significa "extender, esparcir, propagar físicamente” (RAE, 2021c, párr. 1). Otro significado de difundir es "propagar o divulgar conocimientos, noticias, actitudes, costumbres, modas, etc.” (RAE, 2021c, párr. 3).

De acuerdo con las anteriores definiciones, se plantea que la divulgación de la ciencia comienza en el momento que se pone a disposición de cualquier persona y que, además, la información expuesta se espera que comunique hallazgos que en algún momento puedan ser útiles para transformar la realidad del contexto de los seres humanos. Tal como afirmó el exdirector del Museo Nacional de Ciencia y Tecnología (MUNCYT), que al respecto dijo:

Los investigadores deben contar los descubrimientos científicos, el método, los recursos, las actitudes, la relación con otros campos del saber, y tener interés en que "toda persona pueda leerlo”. Esas son razones para seguir teniendo a Galileo como referencia de cultura científica y de divulgación cuatro siglos después. (Núñez, 2010, párr. 7)

Con esta aseveración se aprecia que la idea de que la ciencia esté al alcance de todo tipo de público sigue vigente desde la época de Galileo; además, se pretende que las personas pierdan el temor a lo desconocido o a la incomprensión de temas derivados de diversas disciplinas o descubrimientos que han revolucionado al mundo.

En una publicación del blog del Consejo de Ciencia, Tecnología e Innovación del Estado de Hidalgo (CITNOVA), se describe que: 
La difusión se preocupa porque el mensaje llegue a conocedores del tema, principalmente científicos, investigadores $\mathrm{y}$ especialistas, mientras que la divulgación se propone expandir esos conocimientos a todas las personas interesadas y, para lograrlo, busca que el mensaje sea atractivo, fresco, pero sin que por eso desvirtúe el contenido. Sin divulgación de la ciencia no se puede construir una cultura científica. (CITNOVA, 2021, párr. 4).

Ambos conceptos en apariencia pueden significar lo mismo; sin embargo, existe una sustancial diferencia, que tiene que ver con el tipo de público a quien va dirigida la información y el alcance que se pretende lograr. Ya lo describía Espinosa Santos (2010) de una manera tan clara, cuando dijo "que la difusión es la propagación del conocimiento entre especialistas y constituye un tipo de discurso diferente, contiene un conjunto de elementos o signos propios de un discurso especializado y una estructura que se constituye en factores clave a la hora de su evaluación” (párr. 5).
Mientras que Fourez (1992, citado en Espinosa Santos, 2010) planteó que la divulgación de la investigación científica "consiste en una actividad de relaciones públicas de la comunidad científica que se interesa por mostrar al buen pueblo las maravillas que los científicos son capaces de producir” (párr. 4).

Por su parte Calvo (1997) dijo que, para hablar de divulgación de ciencia, es necesario hablar de dos matices: el primero, que se refiere a la alfabetización científica y el segundo, que se atañe a la popularización de la ciencia, a lo cual expresa:

Que pueden advertirse matices entre una y otra expresión, pues se trata en los dos casos de ayudar al público a superar sus temores sobre la ciencia. Ahora, periodistas, científicos y estudiosos europeos prefieren pensar en alfabetización científica, entendimiento o conocimiento público de la ciencia o cultura científica. En todo caso, la idea es llevar la ciencia al público en general, para atender al requerimiento de información científica y 
ayudar al hombre común a superar sus temores en relación a la ciencia. (p. 4).

El mismo autor expresó que la divulgación de la ciencia tiene dos objetivos: uno, vinculado con el conocimiento, que tiene que ver con comunicar al público los avances de las múltiples disciplinas de nuestro tiempo, como son: biología, economía, ciencias sociales, ciencias administrativas, entre otras. En otras palabras, ayuda a la gente a conocer y comprender su entorno tanto visible como invisible. $Y$ el otro, tiene que ver con los planes de acción llevados a cabo por centros de investigación, universidades e instituciones educativas en general, periodistas, investigadores y docentes (Calvo, 1997, p. 8).

En consecuencia, la difusión de la ciencia se enfoca a un público especializado sobre un tema específico, mientras que la divulgación busca que la información sea comunicada a todo tipo de personas. Una vez que se aprecia la diferencia entre ambos conceptos y para efectos de esta comunicación, en lo sucesivo se hablará de divulgación de la ciencia como el acto de comunicar la ciencia al público en general.

\section{|Aspectos favorables de divulgar la ciencia}

En el blog del Instituto Politécnico Nacional (IPN), Pacheco (2020) refiere que: "la divulgación de la ciencia es un campo multidisciplinario, cuyo objetivo es comunicar, utilizando una diversidad de medios, el conocimiento científico a diversos públicos voluntarios, recreando ese conocimiento con fidelidad y contextualizando para hacerlo accesible” (párr. 5).

Al hablar de públicos voluntarios es que se deja abierta la opción a cualquier persona interesada en conocer algo acerca de un tema en específico, generalmente de rigurosidad científica; por lo tanto, uno de los aspectos esenciales de divulgar la ciencia tiene que ver con acortar ese trecho entre el mundo científico y el resto del mundo que, en la mayoría de los casos, presenta resistencia para adentrarse a este tipo de información. 
Alboukrek (1991, citado en Calvo, 1997, p. 39) mencionó que la divulgación de la ciencia favorece el desarrollo e integración de diversos aspectos, como:

- La generación de un ambiente que estimula la curiosidad por la ciencia y su método.

- Contribuye a despertar la imaginación.

- Cultiva el espíritu de investigación, a la vez que desarrolla la capacidad de observación, claridad de pensamiento y la creatividad.

- Favorece el descubrimiento de vocaciones científicas.

- Propicia una relación más humana con el o los científicos.

- Erradica mitos.

- Abre caminos hacia la participación del desarrollo cultural universal.

En relación a lo anterior, se puede agregar que la divulgación de la ciencia favorece también el desarrollo cultural en una sociedad, pues cuando un pueblo está bien informado con fuentes veraces, entonces la sociedad incrementa el poder de decisión $y$ sus ciudadanos son capaces de combatir la indiferencia ante determinadas situaciones. Así también, contribuye a elevar la calidad de vida al proporcionar a las personas múltiples herramientas que pueden utilizar para aprovechamiento de los recursos.

Si la sociedad tiene acceso a la ciencia a través de su divulgación, entonces es probable que los procesos de enseñanza se vean fortalecidos de manera transversal, pues no todos los conocimientos pueden adquirirse en el contexto educativo; sin duda, un efectivo hábito sería reforzar desde casa y a temprana edad, la lectura de artículos científicos al alcance del entendimiento, que desarrolle en el individuo la curiosidad por acontecimientos que generen cambios positivos en la humanidad. Otro aspecto no menos importante, que tiene que ver con las circunstancias actuales sobre las formas de divulgar la ciencia, es que favorece la comunicación síncrona al hacer uso de distintas aplicaciones digitales y que logran notificar de manera casi instantánea 
los resultados de investigaciones, tal es el caso de las llevadas a cabo para lograr la vacuna contra el COVID-19, cuyas noticias acerca de este tema están al alcance de la comunidad mundial.

\section{|Formas actuales de divulgar la ciencia}

Al finalizar la segunda década del siglo XXI, la divulgación de la ciencia se puede hacer de distintas formas y desde diferentes medios. Básicamente, se puede acceder a la información científica a través de plataformas digitales que integran redes sociales, blogs, páginas web, metabuscadores, entre otros $\mathrm{y}$ desde dispositivos inteligentes, como smartphones, computadoras, tabletas, entre otros medios.

La Fundación Española para la Ciencia y la Tecnología (FECYT) realizó una encuesta de percepción social de la ciencia en el año 2018; los resultados indican que:

La información científica se hace accesible en plataformas como redes sociales, páginas o blogs tomando un rol muy importante, pues el internet y las redes sociales son la principal fuente de información sobre ciencia para las personas de 15 a 44 años (Informador.mx, 2019, párr. 1).

Al hacer uso de la Internet, la divulgación de la ciencia cobra un mayor alcance, propiciando que niños, jóvenes y adultos tengan fácil acceso a diversas comunidades científicas desde sus dispositivos móviles. Además, el contar con cierto nivel de alfabetización digital hace posible que las personas interesadas en ciertos temas puedan generar interacciones en redes sociales, tales como Facebook, Messenger, Twitter, Instagram, WhatsApp, Telegram, YouTube, Snapchat, LinkedIn y muy recientemente Tik Tok, cuya red social es tan popular que se puede encontrar cualquier tipo de información en la misma.

Es así que la difusión y divulgación de la ciencia, han caminado permanentemente junto al desarrollo y actualización constante de las TIC, pues ofrecen la posibilidad de realizar búsquedas específicas y de manera instantánea, generando así la adquisición de conocimientos, ya que: 
Con la evolución de las TIC han surgido otros conceptos que resulta interesante mencionar pues aluden al conocimiento y al aprendizaje de éstas, uno de ellos son las TAC (Tecnologías del Aprendizaje y del Conocimiento) cuyo propósito es relacionar a la tecnología y al conocimiento. Significa entonces, que cuando se utilizan TIC para generar conocimiento desde cualquier ambiente de aprendizaje, se convierten en TAC, debido a que a través de éstas se crea, comparte y difunde toda información relacionada con el manejo de las tecnologías. (Morales Salas \& Rodríguez Pavón, 2020, p. 26).

Ahora bien, en el momento que las personas comienzan a interactuar con el mismo interés de crear, aprender y crecer en distintas áreas de la ciencia y en la búsqueda de soluciones distintas a una misma temática, se forman las llamadas comunidades de aprendizaje y es cuando surge un concepto más que también evoluciona de las TIC y que refiere a la participación de las personas en redes sociales; en este sentido los autores citados anteriormente, señalan que se trata de las Tecnologías de Empoderamiento y Participación (TEP), y que sin duda:

Resultan muy atractivas para el usuario y el momento actual, pues hacen referencia al mundo social y participativo donde se puede trabajar y expresar sin límites; donde no es necesario la presencialidad, sino que puede ser de manera virtual o remota. Esto genera una mayor interacción con el computador, no así con las personas, pues a través de las TEP, sus usuarios son capaces de generar contenidos en una gran, extensa e ilimitada comunidad virtual. (Morales Salas \& Rodríguez Pavón, 2020, p. 27).

Es entonces cuando la investigación científica cobra mayor relevancia, es decir, cuando se divulga a través del Internet, y es precisamente a través de las TIC, TAC y TEP que las distintas comunidades científicas catalizan o impulsan la divulgación de la ciencia a través de revistas y artículos científicos. Estos artículos pueden ser encontrados en múltiples repositorios en acceso abierto y cuya 
función es organizar, resguardar y difundir múltiples producciones documentales temáticas

institucionales; además favorecen la accesibilidad al lector. De esta manera, al aumentar la visibilidad de la información científica, a través de los repositorios, se genera un impacto considerable en la lectura y utilización de dicha información.

Ahora bien, no basta con depositar la información científica en los repositorios, sino que es importante, por un lado, que el público cuente con las competencias digitales necesarias para saber buscar la información pertinente $\mathrm{y}$, por otro, que dichos repositorios estén visibles; y es precisamente que Google Scholar es el buscador más potente para alcanzar la codiciada visibilidad en los trabajos académicos.

De acuerdo con Marquina (2019) el Ranking Web of Repositories publicó el top de los tres repositorios científicos españoles en acceso abierto con el mayor número de registros indexados en Google Scholar, los cuales fueron: a)
Dialnet, con más de 598 mil recursos documentales, entre artículos científicos, libros, actas de congreso, reseñas bibliográficas, obras colectivas, tesis doctorales, entre otros; b) RACO (Revistas Catalanas con Acceso Abierto), con más de 147 mil recursos, y c) Portal de Acceso abierto al Conocimiento de la UPC (UP Commons), con más de $65 \mathrm{mil}$ tipos de recursos.

Asimismo, en México se cuenta con importantes repositorios para divulgar la ciencia; uno de ellos es el Repositorio Nacional, plataforma orientada para poner a disposición del público en general, información científica, tecnológica y de innovación generada principalmente con recursos públicos. Este repositorio cosecha información de distintos repositorios institucionales como son: RAD-UNAM, RI, RITEC, DSpace, Colección digital de la Universidad de Nuevo León, Irekani, Repositorio Institucional de la Universidad Veracruzana, Repositorio Institucional Zaloamatl, Repositorio de la Universidad de las Américas Puebla, REL, entre otros 
más. Éste puede ser consultado en el siguiente enlace: http://www. repositorionacionalcti.mx/

Los anteriores repositorios son solo algunos localizados en países iberoamericanos a los que se puede recurrir. Sin embargo, en la red se pueden encontrar de otros países, con variada y múltiple información, dependiendo del tema y la institución, así como el idioma, el país, entre otros aspectos particulares. Es importante saber elegir el repositorio más adecuado, y una vez que se elige, es esencial discriminar información para seleccionar la más conveniente y de acuerdo con las necesidades de búsqueda, ya que en la red digital es fácil encontrarnos en un laberinto sin fin en la búsqueda de información confiable.

\section{|Ventajas y desventajas de la divulgación de la ciencia en redes sociales}

Si bien, los repositorios se encuentran en la Internet de forma ordenada, pues son webs que contienen grandes cantidades de información, a manera de paquetes, el investigador no debe descartar las redes sociales como medio para divulgar su trabajo, pues éstas son herramientas que fungen como un trampolín no solo para difundir y divulgar, sino para crear y mantener relaciones sobre temas en común o bien de interés público. Las redes sociales tienen enormes ventajas, pero también tienen algunos puntos en contra. Autores como Fernández et al. (2019) describen algunos de ellos que inciden en la divulgación de la ciencia a través de redes sociales, información que se muestra en la tabla 1.

Estos autores realizan una importante clasificación de ventajas y desventajas que tienen los científicos al divulgar la ciencia en redes sociales. Desde mi postura sugiero que una ventaja de particular importancia es la comunicación síncrona y asíncrona que pueden tener los interesados en y desde distintos lugares del planeta, lo que conlleva al aprovechamiento de las TIC, las TAC y las TEP. 
Tabla 1.

Ventajas y desventajas para divulgar ciencia en redes sociales

Ventajas de las redes sociales

Desventajas de las redes sociales

Su sistema de comunicación es inmediato.

Su registro no tiene costo económico.

Facilitan la interacción entre usuarios.

Se convierten en escenario de interesantes debates.

Conectan personas de diferentes lugares del mundo.

Acercan los "grandes científicos” con aquellos que "están empezando”.

Aumentan la cantidad y el tipo del perfil del público al que llegan los resultados de investigación.

Permiten una búsqueda rápida de información.
En ocasiones, impera la rapidez sobre la calidad.

Generan competitividad por ser el primero en difundir una información.

Se convierten en un canal de propagación de bulos o fake news.

A veces, van directas al resultado de la investigación, sin informar de otros aspectos interesantes del proceso.

El acercamiento entre periodismo y ciencia ciudadana puede suponer un peligro para la rigurosidad de la información.

En los debates sin moderador se puede traspasar la barrera del respeto.

Cesión de datos personales de los usuarios a cambio de publicidad.

Nota: Fernández et al. (2019).

\section{| Aportaciones}

Con relación a los aspectos que favorecen la divulgación de la ciencia descritos por Alboukrek (1991, citado en Calvo, 1997), además de lo mencionado por este autor, sugiero otros aspectos que a continuación se describen:

- El divulgar la ciencia sirve para dar a conocer los avances que se originan en múltiples disciplinas.

- Es esencial que la divulgación de la ciencia emane, no solamente desde los recintos escolares, sino también desde la urbe gubernamental; esto significa que los gobiernos deberían proveer a la sociedad de información confiable sobre hallazgos que contribuyan al desarrollo social y tecnológico.

- El que la sociedad tenga cierto nivel de alfabetización científica favorece el aumento de la calidad de vida de los individuos. 
- Los científicos o divulgadores de ciencia deben saber comunicar el proceso y los resultados de sus hallazgos; esto impactaría directamente en eliminar prejuicios por parte del público espectador.

- Es importante fomentar, o bien, inmiscuir al público en general y que así lo desee, a una alfabetización científica, logrando una fusión entre el científico y la sociedad.

El hecho de que el científico o investigador comunique sus hallazgos en redes sociales favorece que se sensibilice acerca de un uso más pensado sobre el tipo de lenguaje puesto a disposición de un público no especializado, permeando así sus conocimientos en las personas que estén dispuestas a leer sus investigaciones. Parece fácil, sin embargo, a la mayoría de los científicos, en ocasiones no les resulta cómodo hablar en lenguaje cotidiano, pues no se trata de hablar o narrar lo que propiamente se hizo en la investigación, sino más bien, debe ser capaz de generar curiosidad e interés en los lectores, oyentes o espectadores.
En relación a las ventajas y desventajas acerca de divulgar la ciencia en redes sociales mencionados por Fernández et al. y otros autores, considero que son más las ventajas, pues se tiene un rápido acceso al conocimiento por parte del lector; por lo tanto, el investigador tiene la oportunidad de explicar los resultados de su investigación de una manera directa.

Otra ventaja es que, al conectarse entre científicos pares, se puede lograr la ejecución de proyectos internacionales. Así también, los beneficios de estos proyectos se divulgan con mayor rapidez, propiciando el cierre de brechas entre el conocimiento y la ausencia de éste.

Permite que los científicos salgan de su mundo inalcanzable para que, a través de la navegación por diversas redes digitales, puedan percatarse de nuevos inventos, fortaleciendo así, los cambios de paradigmas.

Cuando el científico divulga su trabajo, mediante un blog, un post, un tuit o un video subido en YouTube, puede recibir un feedback inmediato a través de un 
comentario, una pregunta, e incluso puede recibir datos que aporten información a su hallazgo divulgado. Este tipo de respuestas inmediatas hace que el científico aprenda a valorar opiniones de un público que no es precisamente erudito en el tema.

Cuando el investigador divulga los resultados en redes sociales propicia las interrelaciones tanto personales como entre especialistas de otros temas que pudieran reforzar el campo de investigación del científico. Sin duda, las enormes ventajas que ofrece la Internet para divulgar la ciencia, no se acabarían de describir en esta comunicación. El aprovechamiento de los medios de comunicación en el siglo XXI, hace que se puedan unir fuerzas y compartir innumerables puntos de vista; pero, así como hay pros, es importante conocer el lado oscuro que conlleva la divulgación de la ciencia y tomar las debidas precauciones.

Otra de las ventajas que es significativo mencionar es que al hacerse más visible el trabajo del investigador, por la facilidad de la conectividad, aumenta su prestigio $\mathrm{y}$ se comienza a posicionar en las redes como experto o líder de determinados temas.

Que el público tenga un clic a la mano, favorece que el trabajo del investigador llegue a públicos internacionales, avanzando hacia nuevas audiencias no exploradas. Así también, al tener alcances internacionales puede llegar a conseguir financiamiento externo, si es el caso.

Lo anterior obedece a algunas de las ventajas importantes que se ciñen en la práctica del investigador al divulgar su trabajo en redes sociales. Sin embargo, una de las desventajas es inexcusablemente la enorme cantidad de información a la que se tiene acceso al momento de entrar en alguna red social. Dicha información no siempre es la más confiable; por lo tanto, optar por seleccionarla y discriminarla es un reto al que se tiene que enfrentar cualquier internauta, ya que debe ser capaz de buscar las fuentes más confiables que le ayuden a tener información precisa, veraz y confiable.

No se debe olvidar que la rigurosidad es un elemento imprescindible en 
la divulgación de la ciencia; sin embargo, en muchas ocasiones ésta no se cumple por algunos usuarios, pues la libertad para crear contenido cibernético en ocasiones destaca la falta de precisión y veracidad en la información. Por lo tanto, no todo lo que se dice en la Internet es verdad.

Parece imposible realizar el trabajo dedicado a verificar la información en la red de redes, pues cada vez se tiene menos contacto con la fuente directa o con los hechos, mermando muchas veces la certeza en lo que se publica.

\section{| Conclusiones}

Ante un escenario de "infoxicación", neologismo que fue acuñado por el especialista en información Alfons Cornella para apuntar a la sobresaturación de información o como acrónimo de intoxicación por información en el año 2013, es importante tener una visión crítica sobre lo que se lee acerca de temas que hablen de ciencia. Seleccionar las fuentes y discriminar aquellas que parecen no ser confiables, son actos imprescindibles que se espera que los internautas hagan.
La divulgación de la ciencia tiene que ver con enlazar un puente entre el mundo de las evidencias científicas y el resto del mundo, propiciando que el público en general integre lo científico a su vida cotidiana, sin olvidar que la ciencia es fáctica, especializada, se basa en un método, es analítica y como tal busca establecer leyes generales.

Divulgar la ciencia es una práctica que lleva no solamente a entender los actos y sus consecuencias sociales, sino que también, ayuda a entender a otros organismos que aun siendo invisibles comparten el ecosistema día a día.

Entre las redes sociales más usadas por los investigadores y científicos para divulgar sus trabajos se pueden aludir a ResearchGate, Methodspace, Academia.edu, DivulgaRed, PlazaScience, Loop, LabRoots, MyScienceWork, Academici, ScholarUniverse, Mendeley, CiteUlike, entre otras. Al identificar estas redes sociales, los científicos o investigadores, por un lado, comienzan a divulgar sus trabajos y conseguir un mayor impacto de visualización, y por otro, si se trata de búsquedas 
realizadas por el público en general como estudiantes, docentes, empresarios, etc., se puede comenzar con búsquedas precisas sobre un determinado tema, alguna metodología o resultado, logrando aminorar la incertidumbre en la búsqueda.

Ahora que han pasado dos décadas desde que inició el siglo XXI, las Tecnologías de Información y Comunicación han tenido una constante y acelerada evolución, transformando procesos en el orden social, político, financiero, educativo y tecnológico. Las grandes bibliotecas que resguardan obras completas como vestigio de descubrimientos que han cambiado al mundo, continúan en la incansable tarea de digitalizar un sinfín de información para ponerla al alcance de cada individuo del planeta. Sin duda, cada vez más, se tendrá acceso a información que antes no estaba visible o bien era imposible consultarla por encontrarse en otros países a los que normalmente no se tiene acceso.
Este es un siglo donde la comunicación abunda y nos invade, aún más cuando en el año 2020 se hizo presente la pandemia provocada por el virus COVID-19 y que millones de personas en todo el mundo tuvieron que aislarse físicamente; este nuevo orden mundial sin duda aceleró las formas de hacer funcionar al mundo desde los ordenadores y los teléfonos inteligentes. Suena irónico decir que el virus nos aisló, pero la tecnología digital nos unió.

Es así que, con el aprovechamiento de las TIC no basta con difundir la ciencia entre pares, en un contexto determinado y con un lenguaje propio de científicos, sino que es esencial hacer llegar la ciencia a todo el público, de manera que ésta sea comprendida, analizada e interpretada de un modo razonable y que sea capaz de transformar la educación y la calidad de vida de los individuos, eso sería el verdadero propósito de la divulgación de la ciencia. 


\section{|Referencias}

Calvo Hernando, M. (1997). Objetivos de la divulgación de la ciencia. Chasqui. Revista Latinoamericana de Comunicación, 60. https://revistachasqui.org/index.php/chasqui/article/view/1153/1182

Cañedo Andalia, R. (1996). Breve historia del desarrollo de la ciencia, ACIMED, 4, 38-41. http://eprints.rclis.org/5364/1/aci07396.pdf

Ciencia. (2020, 12 de diciembre). Qué es ciencia. Significados.com. https://www.significados.com/ciencia/

Consejo de Ciencia, Tecnología e Innovación de Hidalgo [CITNOVA]. (2020, 15 de diciembre). ¿Difusión y divulgación, Qué es? CITNOVA. http://citnova.gob.mx/difusion-y-divulgacion/que-es/

Cornella, A. (2013, 2 de octubre). Infoxicación. Semper Progredi. https://alfonscornella.com/2013/10/02/infoxicacion/

Espinosa Santos, V. (2010). Difusión y Divulgación de la Investigación Científica. Idesia (Arica), 28(3), 5-6. https://dx.doi.org/10.4067/S071834292010000300001

Fernández, I., Menéndez, O., Fuertes, J., Milán, M., \& Mecha, R. (2019). La comunidad científica ante las redes sociales. Guía de actuación para divulgar ciencia a través de ellas. Universidad Complutense de Madrid. https://n9.cl/ob5jw

Informador.mx. (2019, 13 de julio). Las diferentes maneras y medios para comunicar la ciencia. El Informador. https://n9.cl/1at8n

Marquina, J. (2019, 11 de octubre). Los 15 mayores repositorios científicos españoles en acceso abierto según Google Scholar. Acceso Abierto, repositorios. https://n9.cl/b3lgo7 
Morales Salas, R. E., \& Rodríguez Pavón, P. R. (2020). Las competencias digitales en TIC aplicadas en las organizaciones. International Journal of Information Systems and Software Engineering for Big Companies (IJISEBC), 7(1), 25-35. http://www.uajournals.com/ijisebc/en/magazine/ back-issues.html?id=137

Núñez, R. (2010, 30 de marzo). Galileo, pionero de la divulgación científica. El País. https://elpais.com/sociedad/2010/03/30/ actualidad/1269900010_850215.html

Pacheco, M. R. (2020, 18 de febrero). ¿Divulgar la ciencia?, ¿por qué? y ¿para qué? Instituto Politécnico Nacional. https://n9.cl/nebk0

Real Academia Española [RAE]. (2021a). Diccionario de la lengua española. https://dle.rae.es/ciencia

Real Academia Española [RAE]. (2021b). Diccionario de la lengua española. https://dle.rae.es/divulgar

Real Academia Española [RAE]. (2021c). Diccionario de la lengua española. https://dle.rae.es/difundir?m=form 